\title{
Biomassa acima do solo de um ecossistema de "campina" em Roraima, norte da Amazônia Brasileira.
}

\author{
Reinaldo Imbrozio BARBOSA ${ }^{1}$, Carlos Alberto Cid FERREIRA ${ }^{2}$
}

\begin{abstract}
RESUMO
Foi estimada a biomassa (viva + morta) acima do solo de um ecossistema de "campina" localizado em Roraima, norte da Amazônia brasileira. A biomassa foi determinada a partir de um inventário fitossociológico (1 ha amostral) e distribuída em dois estratos: (1) gramíneo-lenhoso, composto de "ervas + liquens" (Poaceae, Cyperaceae, Eriocaulaceae, Cladonia spp), Bromeliaceae, plântulas, "litter" fino e grosso e, (2) arbóreo-arbustivo, composto por árvores e arbustos. O estrato gramíneo-lenhoso foi estimado pelo método direto (corte e pesagem) através de 10 quadras de $1 \mathrm{~m}^{2}$, aproveitando os transectos do inventário. O estrato arbóreo-arbustivo foi estimado pelo método indireto com o corte de 98 indivíduos de diferentes espécies e diâmetros. Foi gerado um modelo para expressar a relação entre a biomassa seca total $(\mathrm{kg})$, a circunferência de base $(\mathrm{cm})$ e a altura total $(\mathrm{m})$ para os indivíduos deste estrato. A equação foi aplicada nos 3.966 indivíduos.ha ${ }^{-1}$ observados no inventário. A biomassa total foi estimada em 15,91 t.ha ${ }^{-1}$, sendo $2,20 \pm 0,23$ t.ha $^{-1}(13,8 \%)$ do estrato gramíneo-lenhoso e 13,70 \pm 7,13 tha ${ }^{-1}$ (86,2\%) do arbóreo-arbustivo. A espécie arbórea de maior biomassa foi Humiria balsamifera (Aubl.) St. Hill. (8,43 t.ha-1 ${ }^{-1}$, seguida de Pagamea guianensis Aubl. (1,14 t.ha $\left.{ }^{-1}\right)$. Estes resultados são importantes para refinar os cálculos de emissão de gases do efeito estufa pela queima e decomposição da biomassa acima do solo em ecossistemas de campinas na Amazônia.
\end{abstract}

\section{PALAVRAS-CHAVE}

campina, biomassa, Roraima, fitomassa

\section{Aboveground biomass of a "campina" ecosystem in Roraima, Northern of Brazilian Amazonia.}

\begin{abstract}
The aboveground biomass of a "campina" ecosystem was estimated in Roraima, in the northern portion of Brazilian Amazonia. The biomass was determined from a phytosociological inventory (1 ha) and distributed between two categories: (1) grassy-woody, composed of "berbs+lichens" (Poaceae, Cyperaceae, Eriocaulaceae, Cladonia spp), Bromeliaceae, seedlings, fine and coarse litter and, (2) woody, composed of trees and bushes. The grassy-woody category was estimated by the direct method (cutting and weighing) in $101-\mathrm{m}^{2}$ plots, taking advantage of transects of the inventory. The category woody was estimated by the indirect method cutting 98 individuals of different species and diameters. A model was generated to express the relationship among total dry above-ground biomass (kg), base circumference $(\mathrm{cm})$ and total height $(\mathrm{m})$ for the individuals in this category. The equation was applied to the 3,966 ind.ha ${ }^{-1}$ observed in the inventory. The total biomass was estimated at 15.91 t.ha $a^{-1}$, of which $2.20 \pm 0.23$ t.ha ${ }^{-1}$ (13.8\%) was in the grassy-woody category and $13.70 \pm 7.13 \mathrm{t} . \mathrm{ha}^{-1}$ (86.2\%) in woody category. The tree species with the bighest biomass was Humiria balsamifera (Aubl.) St. Hill. $\left(8.43\right.$ t.h $\left.a^{-1}\right)$, followed by Pagamea guianensis Aubl. $\left(1.14 t . h a^{-1}\right)$. These results are important for refining the calculations of greenhouse-gas emissions from burning and decomposition of above-ground biomass in "campina" ecosystems in the Amazon region.
\end{abstract}

\section{KEY WORDS \\ campina, biomass, Roraima, phytomass}

\footnotetext{
${ }^{1}$ INPA/CPEC Caixa Postal 96 69301-970 Boa Vista - RR Fone/fax : 0xx-95-623 9433 e-mail : reinaldo@inpa.gov.br

${ }_{2}^{2}$ INPA/CPBO Caixa Postal 478 69083-970 Manaus - AM Fone: 0xx-92-643 3109 e-mail:cacid@inpa.gov.br
} 


\section{ACTA \\ AMAZONICA}

BIOMASSA ACIMA DO SOLO DE UM ECOSSISTEMA DE "CAMPINA"

EM RORAIMA, NORTE DA AMAZÔNIA BRASILEIRA

\section{INTRODUÇÃO}

Estudos sobre a biomassa dos ecossistemas tropicais são de grande interesse ecológico possibilitando estimativas de fluxos e balanços de nutrientes nestes sistemas (Jordan \& Uhl, 1978). Sua quantificação também é função direta para os cálculos de emissão de gases do efeito estufa pela queima e decomposição da matéria orgânica em sistemas naturais e antropizados (Brown \& Lugo, 1992; Fearnside 1992; Fearnside, 1994; Brown, 1997). Sendo assim, as estimativas de biomassa são necessárias para um melhor entendimento dos impactos do desmatamento no aquecimento global (Lu et al., 2002). Na Amazônia Brasileira poucos são os estudos realizados nas diferentes tipologias vegetais. Os estudos existentes são quase que totalmente direcionados para sistemas "florestais", de alta biomassa, por causa do maior impacto nas emissões e das elevadas taxas de desmatamento por toda a região (INPE, 2002). Entretanto, sistemas "nãoflorestais" (baixa biomassa) também estão sofrendo uma acelerada mudança no uso original da terra. Associado à ação do fogo, estas mudanças podem influenciar não só a dinâmica da biomassa vegetal nestes ecossistemas abertos, como também o estoque e a liberação de carbono na forma de gases do efeito estufa (Barbosa, 2001; MCT, 2002).

As campinas amazônicas constituem um ambiente regional com estudos muito fragmentados que acusam baixa diversidade vegetal e alto endemismo (Anderson, 1981). O termo "campina" na região amazônica ainda é muito discutido, mas refere-se, em geral, a uma paisagem caracterizada por vegetação de porte baixo (3-4m), aberta e escleromórfica, que ocorre sempre em solos arenosos de baixa fertilidade, principalmente em áreas de influência da bacia do Rio Negro (Takeuchi, 1960; Sioli, 1960; Rodrigues, 1961, Prance, 1975, Eiten, 1992; Ferreira, 1997). São ambientes que, devido as naturais condições edafológicas, passam por um déficit hídrico extremo nos períodos sem chuva, facilitando a propagação de fogos antropogênicos pelo acúmulo de biomassa seca sobre o solo. Na Amazônia elas correspondem a aproximadamente $34.277 \mathrm{~km}^{2}$, distribuídas entre os estados do Amazonas $\left(22.838 \mathrm{~km}^{2}\right)$ e Roraima (11.439 $\mathrm{km}^{2}$ ), levando em consideração a distribuição espacial dos ecossistemas originais adotada pelo Projeto RADAMBRASIL (Brasil, 1975) e contabilizada por Barbosa \& Fearnside (2002).

A ação do fogo e da troca do uso da terra nas campinas ainda é pouco conhecida. Há um reduzido volume de informações sobre os parâmetros de cálculo que permitem estimar com maior confiança a emissão de gases do efeito estufa pela queima e decomposição da biomassa vegetal nestes sistemas. Estas estimativas são parte integrante da "Convenção Quadro das Nações Unidas sobre Mudança do Clima" de 1992, e acordados internacionalmente no Protocolo de Quioto de dezembro de 1997 (http:// www.mct.gov.br/clima). Neste sentido, o objetivo deste estudo é o de estimar a biomassa vegetal (fitomassa) acima do solo de um ecossistema de campina situado no Estado de Roraima, ampliando a base dos parâmetros de cálculo, e refinando os valores atualmente utilizados nas estimativas de emissão de gases do efeito estufa provenientes da troca do uso da terra dos ecossistemas amazônicos.

\section{MATERIAL E MÉTODOS}

\section{Área de Estudo}

A área de estudo fica situada no extremo norte da Amazônia brasileira, próxima do entroncamento da rodovia estadual RR 170 com a vicinal 1 da Colônia Agrícola da Confiança II, Município de Cantá, Roraima, aproximadamente $02^{\circ} 29^{\prime} 11^{\prime \prime} \mathrm{N}$ e $60^{\circ} 38^{\prime} 04^{\prime \prime} \mathrm{W}$ (Datum SAD 69). A topografia do terreno é plana e o solo é arenoso (podzol), típico das campinas amazônicas. Vários tufos de liquens Cladonia spp são facilmente percebidos sobre o solo, sendo que a espécie arbórea de maior evidência é Humiria balsamifera (Aubl.) St. Hill. (Humiriaceae). A pluviometria da localidade é de $2.090 \mathrm{~mm} \cdot$ ano $^{-1}$, levando em consideração os dados do Campus Experimental da Confiança III da Embrapa Roraima (1995 a 2002) situado à aproximadamente $25 \mathrm{~km}$ de distância (Mourão et al., 2003). O pico da seca se estende de dezembro a março e, o das chuvas, de maio a agosto. Não existem dados de umidade relativa do ar e temperatura média de localidades próximas. Seguindo a classificação tipológica do Projeto RADAMBRASIL (Brasil, 1975), ampliada e revisada pelo IBGE (1992), esta vegetação é uma forma de "campinarana (campina) arborizada", de codificação "La". Neste tipo de ambiente, são comuns manchas de vegetação arbóreoarbustiva associadas a áreas limpas, com tufos de material gramíneo-lenhoso (Figura 1). Em Roraima, a campina investigada é considerada uma área de borda, situada no início do complexo do interflúvio Rio Branco - Rio Negro, onde se encontram os grandes blocos de ecossistemas de campinas e campinaranas do extremo norte amazônico.

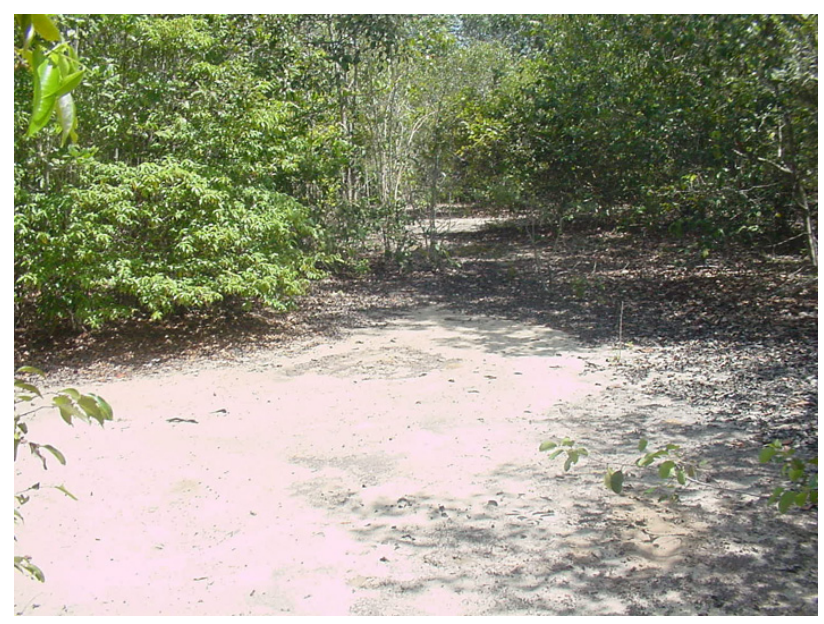

Figura 1 - Aspecto geral do ecossistema de campina da região da Confiança II, Município do Cantá, Roraima. 


\section{ACTA \\ AMAZONICA}

BIOMASSA ACIMA DO SOLO DE UM ECOSSISTEMA DE "CAMPINA" EM RORAIMA, NORTE DA AMAZÔNIA BRASILEIRA

\section{Amostragem da Biomassa}

Toda a amostragem da biomassa foi realizada entre $20.11 \mathrm{e}$ 15.12.2002. Neste trabalho, estamos considerando o termo "biomassa" como o conjunto dos elementos vegetais, vivos e mortos, presentes acima do solo no ambiente de campina. Para tanto, a biomassa foi dividida em dois estratos (categorias) para facilitar o entendimento de seu fracionamento:

\section{(1) Gramíneo-lenhoso}

Este grupo foi definido como representante das seguintes frações: (a) "litter" fino (folhas secas, necromassa de madeira com peças de diâmetro $\ddot{i}$ $2,0 \mathrm{~cm}$ e todos os restos orgânicos depositados sobre o solo), (b) "litter" grosso (necromassa de madeira, em pé ou tombada, com diâmetro $>2,0 \mathrm{~cm}$ ), (c) Bromeliaceae (indivíduos da família das bromélias), (d) "ervas + liquens" (Poaceae, Cyperaceae, Eriocaulaceae, Cladonia spp) e (e) plântulas (jovens de espécies arbóreo-arbustivas com circunferência de base $<5 \mathrm{~cm}$ ou diâmetro $<1,6 \mathrm{~cm}$ ). Circunferência de base $(\mathrm{Cb})$ é definida neste estudo como o comprimento da circunferência do caule de cada indivíduo arbóreoarbustivo observado na altura média entre a linha do solo e o primeiro galho representativo de cada indivíduo ou de suas ramificações, separadamente. Isto foi necessário porque muitas espécies de árvores e arbustos das campinas possuem grande número de ramificações, sendo inapropriado fazer uso de padrões adotados para florestas (DAP à $1,30 \mathrm{~m}$ de altura) ou cerrados arborizados $(0,30 \mathrm{~m}$ de altura), ou mesmo medidas de circunferência próximas ao solo, que não representariam adequadamente o ambiente das campinas.

O método de coleta deste material foi o direto (corte e pesagem) e consistiu em estabelecer 10 quadras de $1 \mathrm{~m}^{2}$ dispostas, pelo menos, a 50m de distância entre elas. Isto foi feito aproveitando a espacialização e a linha central dos "transectos" do inventário fitossociológico que estava sendo realizado no mesmo período por C. A. C. Ferreira, em 20 parcelas de $500 \mathrm{~m}^{2}$ cada uma, distribuídas pela área da campina. Todo o material encontrado nas quadras foi pesado e classificado dentro das frações préestabelecidas. Uma amostra de cada fração foi coletada para determinação da umidade (U\%) e do peso seco total, por unidade amostral.

Para determinação da U\%, todas as amostras das frações deste estrato foram secas em estufa $\left( \pm 65^{\circ} \mathrm{C}\right)$ até peso seco constante. O resultado foi obtido da seguinte forma:

$$
\begin{gathered}
\mathrm{U} \%=\text { Pua }- \text { Psa } \times 100 \\
\text { Pua }
\end{gathered}
$$

Onde Pua = peso úmido da amostra em gramas (g), Psa = peso seco da amostra em gramas (g) e U\% é a porcentagem de umidade presente em cada fração avaliada.
O peso seco de cada fração (por quadra e total) foi obtido através da subtração da massa correspondente a U\% do peso úmido total observado em campo.

\section{(2) Arbóreo-arbustivo}

Este grupo é formado pelos indivíduos lenhosos (árvores e arbustos), com circunferência de base (Cb) igual ou superior a $5 \mathrm{~cm}$ ( $\ddot{\mathrm{y}} 1,6 \mathrm{~cm}$ de diâmetro). Ramificaçõos próximas da linha do solo foram consideradas individualmente.

Para determinação da biomassa deste grupo, procedeuse a duas etapas de trabalho. Primeira, corte de 98 indivíduos distribuídos entre as principais espécies e classe $\mathrm{de} \mathrm{Cb}$, a partir das informações preliminares recolhidas pela equipe do inventário fitossociológico. Esta fase foi importante para ajustar a "seleção do tamanbo da amostra" segundo a metodologia sugerida por Brower et al. (1998). Seguindo o cálculo sugerido por estes autores, nós assumimos um erro padrão da estimativa $\left(\mathrm{S}_{\mathrm{x}}\right)$ aceitável da ordem de $20-30 \%$, devido ao elevado número de indivíduos que seria necessário amostrar para reduzir este valor, por exemplo, para menos de 10\%, (mais de 300 indivíduos). Isso inviabilizaria o trabalho de campo.

A escolha dos indivíduos para a amostragem foi sistematizada dentro das parcelas amostrais do inventário fitossociológico, seguindo o critério de se coletar cada espécie, sob diferentes diâmetros, dentro de sua densidade aproximada determinada pelo inventário. A idéia era a de equilibrar as amostras realizadas para determinação da biomassa com os resultados obtidos na fitossociologia. Isto foi importante no sentido de se atribuir peso (ponderação) amostral às coletas, considerando que as principais espécies (maior densidade) e diâmetros estivessem sendo representados efetivamente dentro da amostragem.

As variáveis hipsométricas observadas para cada um destes indivíduos foram, circunferência de base $(\mathrm{Cb})$ e altura total $(\mathrm{Ht})$, associadas ao peso úmido total $(\mathrm{Pu})$ das "folhas" (folhas, frutos e flores) e da "madeira" (troncos, galhos e gravetos). O Pu foi obtido através da pesagem do material vegetal lenhoso em campo. Para determinação da umidade (U\%) de cada fração arbóreoarbustiva foi tomada uma amostra conjunta de folhas e peças de madeira, que foram secas em estufa $\left( \pm 65^{\circ} \mathrm{C}\right.$ e $\pm 100^{\circ} \mathrm{C}$, respectivamente) até peso seco constante. $\mathrm{O}$ resultado da $\mathrm{U} \%$ foi obtido da mesma forma que o anterior. Com o peso seco total individual associado às variáveis hipsométricas, procedeu-se à segunda etapa de trabalho, que visou a montagem de um modelo de regressão para ser aplicado em cada indivíduo resultante da amostragem do inventário fitossociológico. O histórico de cada modelo testado foi originado dos trabalhos de Overman et al. (1994), Alves et al. (1997) e Higuchi et al. (1998), todos tentando evoluir estimativas para biomassa de florestas na Amazônia. 


\section{Coletas Botânicas}

A coleta do material botânico realizada entre 20 e 30 de novembro de 2002, sempre visando a coleta de indivíduos com a parte reprodutiva presente. Quando o indivíduo se encontrava fértil, eram coletadas 5 (cinco) amostras e, quando estéril, apenas 3 (três). Após as coletas de campo, todo material botânico foi processado na Coordenação de Botânica do INPA, sendo desidratado, triado e identificado. Os exemplares férteis foram incorporados ao Herbário do INPA. A correção dos nomes científicos (família, gênero e espécie) e dos autores foi feito com auxilio de literatura especializada para a região e o pelo "site" www mobot.org. O inventário fitossociológico foi realizado no mesmo período.

\section{RESULTADOS}

\section{Estrutura da Vegetação e Composição das Espécies Arbóreo-arbustivas}

O inventário das espécies arbóreo-arbustivas, com circunferência de base $(\mathrm{Cb})_{\mathrm{i}}^{\mathrm{Y}} 5 \mathrm{~cm}$, determinou um total de 3.966 ind.ha- ${ }^{-1}$, ocupando uma área basal (AB) de 5,898 $\mathrm{m}^{2}$.ha ${ }^{-1}$ (Tabela 1). A maioria dos indivíduos $(89,5 \%)$ se concentrou nas classes de $\mathrm{Cb}<20 \mathrm{~cm}(<6,5 \mathrm{~cm}$ de diâmetro). Apenas uma árvore alcançou $\mathrm{Cb}=71 \mathrm{~cm}$, sendo que o somatório daquelas com $\mathrm{Cb}$ acima de $30 \mathrm{~cm}(\mathrm{n}=57)$ representou somente $1,44 \%$ do total amostrado.

Foram identificadas 21 famílias, distribuídas por 27 gêneros e 34 espécies entre todos os indivíduos amostrados. Dentro deste universo, as principais espécies observadas foram Humiria balsamifera (Aubl.) St. Hill., com $60,4 \%$ de valor de cobertura (VC), seguida de Pagamea guianensis Aubl. e Cybianthus fulvopulverulentus (Mez) Agostini (Tabela 2). Em conjunto, estas três espécies totalizam $79,2 \%$ de VC, dominando a estrutura e a composição do ecossistema. A família de maior expressão foi Humiriaceae com $62,8 \%$ de VC. A Cb média para todo o levantamento foi de $12,4 \pm 5,7$ (DP) cm. Esta variável alcançou a maior expressão na espécie Qualea retusa Spruce ex Warm. $(29,5 \mathrm{~cm})$. A altura total $(\mathrm{Ht})$ média foi de $3,2 \pm 1,3 \mathrm{~m}$, sendo que a espécie com maior Ht foi Emmotum nitens Benth. $(7,5 \mathrm{~m})$.

Tabela 1 - Riqueza (R), número de indivíduos (N), área basal (AB) e classe de cicunferência de base $(\mathrm{Cb})$

\begin{tabular}{|c|c|c|c|c|c|c|c|c|c|c|c|}
\hline \multirow[t]{3}{*}{ Parcela } & \multirow[t]{3}{*}{$R(1)$} & \multirow[t]{3}{*}{$\mathbf{N}$} & \multirow{3}{*}{$\begin{array}{c}\mathrm{AB} \\
\mathrm{m}^{2}\end{array}$} & \multicolumn{8}{|c|}{ Classe de Circunferência de Base (Cb) } \\
\hline & & & & \multicolumn{8}{|l|}{$\mathrm{cm}$} \\
\hline & & & & 5-10 & $10-15$ & $15-20$ & $20-25$ & $25-30$ & $30-35$ & $35-40$ & $>40$ \\
\hline 1 & 6 & 114 & 0,190 & 40 & 33 & 24 & 11 & 4 & 2 & 0 & 0 \\
\hline 2 & 2 & 361 & 0,408 & 177 & 128 & 42 & 10 & 3 & 0 & 0 & 1 \\
\hline 3 & 2 & 168 & 0,299 & 34 & 62 & 50 & 15 & 6 & 1 & 0 & 0 \\
\hline 4 & 14 & 312 & 0,381 & 128 & 113 & 48 & 18 & 4 & 1 & 0 & 0 \\
\hline 5 & 5 & 203 & 0,282 & 82 & 66 & 38 & 10 & 5 & 1 & 1 & 0 \\
\hline 6 & 0 & 75 & 0,122 & 19 & 34 & 14 & 4 & 1 & 2 & 1 & 0 \\
\hline 7 & 0 & 106 & 0,137 & 43 & 39 & 17 & 4 & 3 & 0 & 0 & 0 \\
\hline 8 & 2 & 106 & 0,104 & 73 & 24 & 6 & 0 & 1 & 1 & 0 & 1 \\
\hline 9 & 3 & 117 & 0,158 & 55 & 34 & 19 & 6 & 1 & 0 & 2 & 0 \\
\hline 10 & 0 & 40 & 0,077 & 10 & 12 & 14 & 1 & 2 & 0 & 1 & 0 \\
\hline 11 & 0 & 193 & 0,362 & 33 & 78 & 52 & 21 & 7 & 1 & 1 & 0 \\
\hline 12 & 0 & 290 & 0,491 & 75 & 110 & 63 & 29 & 12 & 1 & 0 & 0 \\
\hline 13 & 0 & 202 & 0,298 & 74 & 77 & 29 & 11 & 5 & 5 & 1 & 0 \\
\hline 14 & 0 & 244 & 0,493 & 81 & 78 & 41 & 17 & 14 & 6 & 5 & 2 \\
\hline 15 & 0 & 193 & 0,265 & 85 & 52 & 36 & 16 & 3 & 1 & 0 & 0 \\
\hline 16 & 0 & 157 & 0,285 & 40 & 47 & 49 & 14 & 5 & 2 & 0 & 0 \\
\hline 17 & 0 & 267 & 0,373 & 121 & 74 & 46 & 18 & 5 & 3 & 0 & 0 \\
\hline 18 & 0 & 313 & 0,430 & 123 & 116 & 44 & 21 & 4 & 4 & 1 & 0 \\
\hline 19 & 0 & 166 & 0,243 & 94 & 32 & 21 & 7 & 7 & 3 & 0 & 2 \\
\hline 20 & 0 & 339 & 0,500 & 140 & 111 & 51 & 17 & 16 & 3 & 1 & 0 \\
\hline Total (ha) & 34 & 3966 & 5,898 & 1527 & 1320 & 704 & 250 & 108 & 37 & 14 & 6 \\
\hline$\%$ & - & - & - & 38,5 & 33,3 & 17,8 & 6,3 & 2,7 & 0,9 & 0,4 & 0,2 \\
\hline
\end{tabular}

(1) $\mathrm{R}$ = número de espécies novas por parcela e $\mathrm{N}$ = número de indivíduos presentes em cada parcela 


\section{ACTA \\ AMAZONICA \\ BIOMASSA ACIMA DO SOLO DE UM ECOSSISTEMA DE "CAMPINA" \\ EM RORAIMA, NORTE DA AMAZÔNIA BRASILEIRA}

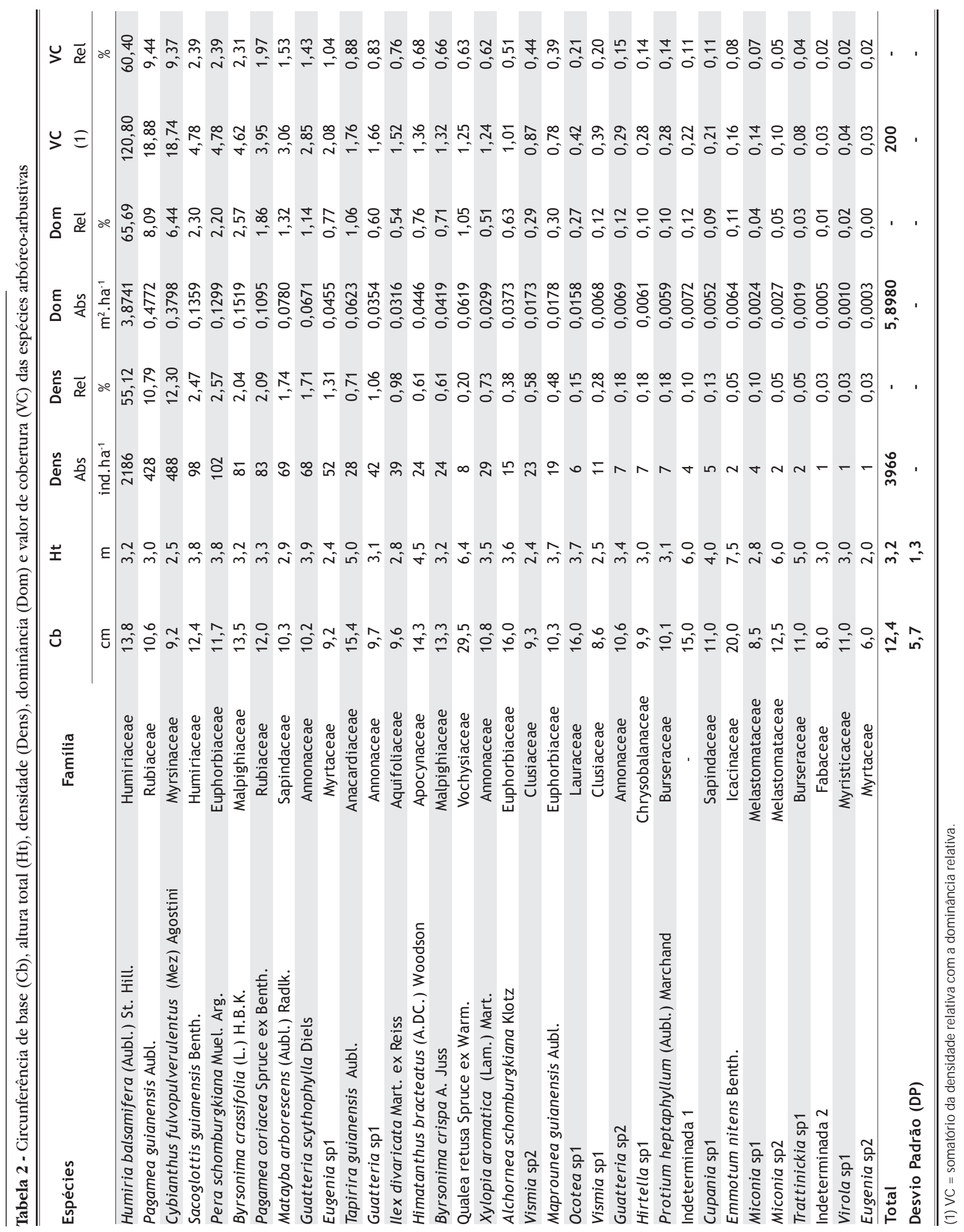




\section{ACTA \\ AMAZONICA}

BIOMASSA ACIMA DO SOLO DE UM ECOSSISTEMA DE "CAMPINA" EM RORAIMA, NORTE DA AMAZÔNIA BRASILEIRA

\section{Modelo de Regressão (Arbóreo-arbustiva)}

Dos 98 indivíduos coletados para a determinação da relação entre a biomassa e os parâmetros hipsométricos, 55 (56,1\%) foram de H. balsamifera (Aubl.) St. Hill., 11 $(11,2 \%)$ de C. fulvopulverulentus (Mez.) Agostini e 8 (8,2\%) de $P$. guianensis Aubl.; o restante (24) foi destinado as demais espécies representando, de forma similar, a distribuição quantitativa determinada pelo inventário fitossociológico. As funções matemáticas que melhor expressam as relações entre a biomassa seca acima do solo dos indivíduos arbóreo-arbustivos e as variáveis circunferência de base e altura total estão apresentadas na Tabela 3. Todas as equações de regressão possuem parâmetros significativos $(\mathrm{P}<0,0001)$. Entretanto, a que possui os melhores indicadores $\left(\mathrm{S}_{\mathrm{x}}\right.$ - erro padrão da estimativa e R2 - coeficiente de determinação) é o Modelo 1 (Figura 2). Este modelo foi usado na computação geral da fitomassa arbóreo-arbustiva acima do solo por espécie e unidade de área.

\section{Biomassa Total Acima do Solo}

A biomassa total acima do solo foi estimada em 15,91 t.ha ${ }^{-1}$ (Tabela 4). Deste total, 13,8\% (2,20 $\pm 0,23$ t.ha $\left.^{-1}\right)$ representam o estrato gramíneo-lenhoso e $86,2 \%$ (13,70 \pm 7,13 t.ha ${ }^{-1}$ ) o arbóreo-arbustivo. O componente de maior participação no grupo gramíneo-lenhoso foi "litter" fino com $1,13 \pm 0,15$ t.ha $^{-1}$ (51,4\%), seguido de "ervas + liquens", com $0,34 \pm 0,06$ t.ha' t $^{-1}(15,5 \%)$. A biomassa viva acima do solo totalizou 14,54 t.ha $^{-1}(91,4 \%)$ e a morta 1,37 t.ha ${ }^{-1}$ $(8,6 \%)$. Do total representado pelo grupo arbóreo-arbustivo, $88,0 \%$ correspondem a fração "madeira" e 12,0\% a de "folhas" (Tabela 5). A biomassa média para cada indivíduo é de $3,45 \mathrm{~kg}$

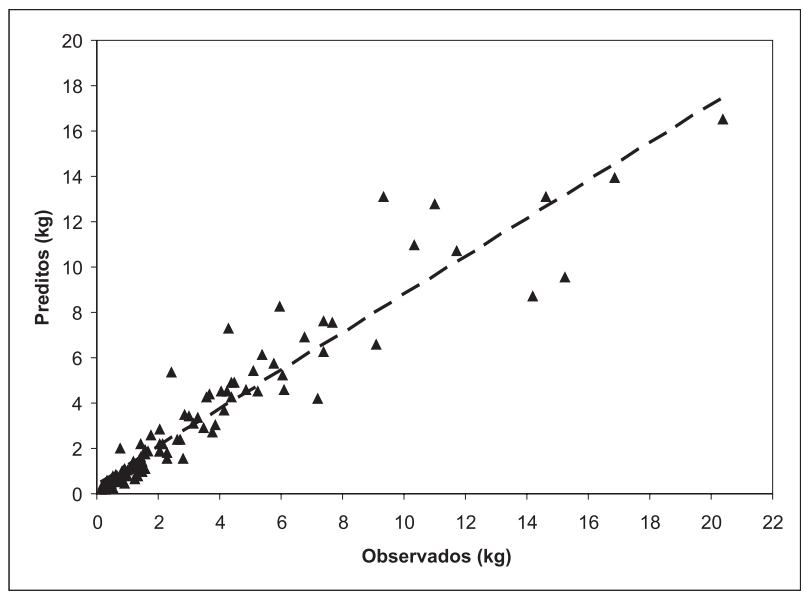

Figura 2 - Tendência central entre os valores "observados" e "preditos" pelo Modelo 1 .

Tabela 3 - Modelos de regressão para estimativa de biomassa acima do solo em sistemas de campina da região da Confiança II, no Estado de Roraima (Bs = biomassa seca total, $\mathrm{Cb}=$ circunferência de base, $\mathrm{Ht}=$ altura total, $\mathrm{R} 2=$ coeficiente de determinação)

\begin{tabular}{|c|c|c|c|c|c|}
\hline \multirow[t]{2}{*}{$\mathrm{N}^{\circ}$} & \multirow[t]{2}{*}{ Modelos } & \multicolumn{2}{|c|}{ Coeficientes } & \multirow{2}{*}{$\begin{array}{c}\text { Erro Padrão da } \\
\text { Estimativa }\end{array}$} & \multirow[t]{2}{*}{ R2 } \\
\hline & & Simbolo & Valor & & \\
\hline \multirow[t]{3}{*}{1} & $\ln B s=a+b \cdot \ln \left(\mathrm{Cb}^{2} \cdot \mathrm{Ht}\right)+\mathrm{c} \cdot(\ln (\mathrm{Cb} 2 \cdot \mathrm{Ht}))^{2}$ & $\mathrm{a}$ & $-3,9041$ & 0,306 & 0,928 \\
\hline & & $\mathrm{b}$ & 0,4658 & & \\
\hline & & $\mathrm{C}$ & 0,0458 & & \\
\hline \multirow[t]{3}{*}{2} & $\ln B s=a+b \cdot \ln \left(C b^{2} \cdot H t\right)+c \cdot C b$ & $\mathrm{a}$ & $-5,0170$ & 0,304 & 0,927 \\
\hline & & $\mathrm{b}$ & 0,8707 & & \\
\hline & & C & 0,0309 & & \\
\hline \multirow[t]{2}{*}{3} & $\ln B s=a+b \cdot \ln \left(C b^{2} \cdot H t\right)$ & $\mathrm{a}$ & $-5,4920$ & 0,309 & 0,925 \\
\hline & & $\mathrm{b}$ & 1,0141 & & \\
\hline \multirow[t]{2}{*}{4} & $\mathrm{Bs}=\mathrm{a}+\mathrm{b} \cdot\left(\mathrm{Cb^{2 }} \cdot \mathrm{Ht}\right)$ & a & $-0,3789$ & 0,332 & 0,888 \\
\hline & & $\mathrm{b}$ & 0,0054 & & \\
\hline 5 & $\ln B s=a+b \cdot \ln (C b \cdot H t)$ & a & $-4,9597$ & 0,388 & 0,883 \\
\hline
\end{tabular}

Tabela 4 - Biomassa total acima do solo (t.ha-1) em um ecossistema de campina de Roraima.

\begin{tabular}{lcccccccc}
\hline \hline \multirow{2}{*}{ Categoria } & Litter & \multicolumn{3}{c}{ Não-lenhosa } & \multicolumn{3}{c}{ Lenhosa (1) } & \multicolumn{2}{c}{ Total } \\
\cline { 2 - 9 } & Fino & Grosso & Bromeliaceae & Ervas+Liquens & Plântulas & Árvores & t.ha $^{-1}$ & $\%$ \\
\hline Gramíneo-lenhosa & 1,133 & 0,236 & 0,225 & 0,341 & 0,267 & - & 2,20 & 13,8 \\
Arbóreo-arbustiva & - & - & - & - & - & 13,70 & 13,70 & 86,2 \\
Total & 1,133 & 0,236 & 0,225 & 0,341 & 0,267 & 13,703 & 15,91 & 100 \\
\hline \hline
\end{tabular}

(1) 0 número de plântulas médio foi de 11.000 por hectare, com um desvio padrão de 1.100. 


\section{ACTA AMAZONICA}

\section{DISCUSSÃO}

As espécies arbóreo-arbustivas determinadas pelo levantamento fitossociológico indicam uma forte tendência monodominante para a campina estudada em Roraima, levando em consideração o elevado valor de cobertura (> $60 \%$ ) de H. balsamifera (Aubl.) St. Hill. Esta espécie é pouco representativa em outras campinas da Amazônia brasileira, como por exemplo, a da "Reserva Biológica de Campina do INPA-SUFRAMA", próxima de Manaus (Anderson et al. 1975). Naquela localidade, ela representa menos de $4 \%$ do índice de valor de importância (IVI) dentre as espécies arbóreoarbustivas observadas (Ferreira, 1997). Rodrigues (1961) não observou nenhum indivíduo de H. balsamifera (Aubl.) St. Hill. entre as principais espécies arbóreas presentes em seus estudos nas "catingas" do rio Negro.

Tabela 5 - Biomassa arbóreo-arbustiva acima do solo das diferentes espécies de uma campina de Roraima.

\begin{tabular}{|c|c|c|c|c|c|}
\hline Espécie & Família & $\begin{array}{c}\text { Madeira } \\
\mathrm{kg} \cdot \mathrm{ha}^{-1}\end{array}$ & $\begin{array}{l}\text { Folhas } \\
\text { kg.ha-1 }\end{array}$ & $\begin{array}{c}\text { Total (1) } \\
\text { kg.ha-1 }\end{array}$ & $\%$ \\
\hline Humiria balsamifera (Aubl.) St. Hill. & Humiriaceae & 7402,32 & 1024,32 & 8426,63 & 61,49 \\
\hline Pagamea guianensis Aubl. & Rubiaceae & 1077,95 & 59,88 & 1137,82 & 8,30 \\
\hline Cybianthus fulvopulverulentus (Mez) Agostini & Myrsinaceae & 574,34 & 55,88 & 630,22 & 4,60 \\
\hline Sacoglottis guianensis Benth. & Humiriaceae & 463,85 & 77,28 & 541,13 & 3,95 \\
\hline Pera schomburgkiana Muel. Arg. & Euphorbiaceae & 365,24 & 55,37 & 420,61 & 3,07 \\
\hline Byrsonima crassifolia (L.) H.B.K. & Malpighiaceae & 310,39 & 64,28 & 374,67 & 2,73 \\
\hline Pagamea coriacea Spruce ex Benth. & Rubiaceae & 318,70 & 48,31 & 367,01 & 2,68 \\
\hline Matayba arborescens (Aubl.) Radlk. & Sapindaceae & 195,80 & 33,51 & 229,31 & 1,67 \\
\hline Guatteria scythophylla Diels & Annonaceae & 196,52 & 29,79 & 226,31 & 1,65 \\
\hline Eugenia sp1 & Myrtaceae & 182,19 & 37,73 & 219,92 & 1,60 \\
\hline Tapirira guianensis Aubl. & Anacardiaceae & 183,90 & 22,22 & 206,12 & 1,50 \\
\hline Guatteria sp1 & Annonaceae & 120,78 & 32,68 & 153,45 & 1,12 \\
\hline Ilex divaricata Mart. ex Reiss & Aquifoliaceae & 102,11 & 23,86 & 125,97 & 0,92 \\
\hline Himatanthus bracteatus (A.DC.) Woodson & Apocynaceae & 93,22 & 13,01 & 106,23 & 0,78 \\
\hline Byrsonima crispa A. Juss & Malpighiaceae & 66,73 & 10,12 & 76,85 & 0,56 \\
\hline Qualea retusa Spruce ex Warm. & Vochysiaceae & 64,69 & 9,81 & 74,50 & 0,54 \\
\hline Xylopia aromatica (Lam.) Mart. & Annonaceae & 61,33 & 8,56 & 69,89 & 0,51 \\
\hline Alchornea schomburgkiana Klotz & Euphorbiaceae & 59,06 & 8,95 & 68,01 & 0,50 \\
\hline Vismia sp2 & Clusiaceae & 44,20 & 6,70 & 50,90 & 0,37 \\
\hline Maprounea guianensis Aubl. & Euphorbiaceae & 34,72 & 2,07 & 36,80 & 0,27 \\
\hline Ocotea sp1 & Lauraceae & 28,56 & 4,33 & 32,89 & 0,24 \\
\hline Vismia sp1 & Clusiaceae & 22,44 & 3,40 & 25,84 & 0,19 \\
\hline Guatteria sp2 & Annonaceae & 20,61 & 3,12 & 23,73 & 0,17 \\
\hline Hirtella sp1 & Chrysobalanaceae & 12,20 & 1,85 & 14,05 & 0,10 \\
\hline Protium heptaphyllum (Aubl.) Marchand & Burseraceae & 11,41 & 1,73 & 13,14 & 0,10 \\
\hline Indeterminada 1 & - & 8,99 & 1,36 & 10,35 & 0,08 \\
\hline Cupania sp1 & Sapindaceae & 8,87 & 1,35 & 10,22 & 0,07 \\
\hline Emmotum nitens Benth. & Icacinaceae & 8,45 & 1,28 & 9,73 & 0,07 \\
\hline Miconia sp1 & Melastomataceae & 7,74 & 1,17 & 8,92 & 0,07 \\
\hline Miconia sp2 & Melastomataceae & 4,54 & 0,69 & 5,23 & 0,04 \\
\hline Trattinickia sp1 & Burseraceae & 3,36 & 0,51 & 3,86 & 0,03 \\
\hline Indeterminada 2 & Fabaceae & 1,34 & 0,20 & 1,54 & 0,01 \\
\hline Virola sp1 & Myristicaceae & 0,72 & 0,11 & 0,83 & 0,01 \\
\hline Eugenia sp2 & Myrtaceae & 0,30 & 0,04 & 0,34 & 0,00 \\
\hline Total & & 12057,6 & 1645,5 & 13703,0 & 100 \\
\hline$\%$ & & 88,0 & 12,0 & - & - \\
\hline
\end{tabular}

(1) números em itálico (madeira / folhas) foram estimados pela média dos demais valores medidos em campo. 


\section{ACTA \\ AMAZONICA}

BIOMASSA ACIMA DO SOLO DE UM ECOSSISTEMA DE "CAMPINA"

EM RORAIMA, NORTE DA AMAZÔNIA BRASILEIRA
A campina de Roraima fica situada em uma área de colonização humana e, por isso, sofre a ação eventual de fogo devido a queimadas fora de controle que penetram no ecossistema. Isto é percebido pelas marcas nas árvores e por pequenas peças de carvão visualizadas sobre o solo. A monodominância de $H$. balsamifera (Aubl.) St. Hill. pode sugerir um ambiente natural diferenciado devido à sua localização de borda amazônica ou pode ser o reflexo de uma ação antropogênica adjacente que acaba influenciando na composição e na estrutura desta campina. Entretanto, a atual recorrência de fogos em ecossistemas de vegetação aberta, como no caso de campinas e cerrados, mesmo fora do controle desta investigação, acaba sendo um fator de manutenção da atual tipologia estrutural, sendo aquela constatada nos sistemas de classificação da vegetação, e utilizada neste estudo como parâmetro para identificação da paisagem dominante. Como as medidas de campo foram efetuadas dois meses antes das queimadas regulares na região, estimamos que os resultados representem o ecossistema em uma situação pré-queima real, tanto para o componente gramíneo-lenhoso (o mais afetado por fogos), quanto para o arbóreo-arbustivo (menos afetado).

Os resultados aqui expressos para distribuição de classes de diâmetro, área basal e ausência de árvores grandes representam um ecossistema de campina arborizada (La), e se identificam com o que determina o sistema de classificação brasileiro editado pelo IBGE (1992). Em termos comparativos, a biomassa acima do solo encontrada para esta campina de Roraima $(15,91$ t.ha-1 $)$, é pouco mais que o dobro do ambiente "Open Bana" (campina aberta - 7,73 t.ha' ${ }^{-1}$ e, inferior por um fator de 4,5 a "Low Bana" (campina baixa - 71,61 t.ha' ${ }^{-1}$ ), determinados em San Carlos do Rio Negro, Venezuela, por Bongers et al. (1985) (Tabela 6). Bongers e colaboradores dividiram as feições de campinas/ campinaranas (Banas) de seu local de estudo em seções horizontais e isoladas (de pequena área amostral), entendendo que seria o melhor caminho para aplicar o método direto (destrutivo), e obter a biomassa acima do solo. Entretanto, "Open Bana" são as manchas de vegetação gramíneo-lenhosa e "Low Bana" são as manchas de vegetação arbóreo-arbustiva que compõe o conjunto das campinas arborizadas (La) pelo sistema de classificação brasileiro. Esta diferenciação tornou as comparações pouco apropriadas com o nosso estudo. Os transectos que nós utilizamos no inventário $(10 \mathrm{~m}$ x $50 \mathrm{~m})$ cortavam ambos os ambientes, considerando este conjunto uma única paisagem conforme IBGE (1992). Logo, independente do método adotado para cálculo da biomassa (direto ou indireto), os dois trabalhos analisam o mesmo ambiente por um prisma diferente. Os demais trabalhos listados na Tabela 6 fazem parte de ambientes tipicamente florestados (campinaranas) como, por exemplo, o de Klinge \& Herrera (1983) ou não possuem dados de biomassa acima do solo, como o de Klinge (1973). Entretanto, são trabalhos pioneiros que ajudam na demonstração das diferenças estruturais entre ecossistemas de campinas e campinaranas amazônicas.
Apesar do choque de métodos entre os valores determinados para Roraima e Venezuela, ambos apontam para uma grande diferença daqueles usados por Fearnside (1997) no sentido de calcular a emissão de gases do efeito estufa pela queima e decomposição da biomassa acima do solo em ambientes de campinas amazônicas de tipologia La. Os números utilizados por Fearnside (1997) são resultado de um conjunto de relações que envolvem a transformação de dados volumétricos derivados do Projeto RADAMBRASIL em biomassa (Brown \& Lugo, 1992; Fearnside, 1992). Nesta argumentação, o sistema de campina La (não-florestal) foi calculado como florestal e com uma biomassa total (acima + abaixo) da ordem de 435 t.ha ${ }^{-1}$ para os limites da Amazônia Legal Brasileira. Isto resulta em uma biomassa acima do solo estimada em 178 t.ha-1 (41\% do total), um valor superior por um fator de 11,2 vezes do que o estimado para a campina de Roraima $(15,91$ t.ha $\left.{ }^{1}\right)$. Levando em consideração os dados da Tabela 6 e os parâmetros de cálculo estipulados por Fearnside (1997), é possível supor que apenas para o ambiente La há aproximadamente $0,49 \times 10^{6}$ t de biomassa (acima + abaixo do solo) contabilizados em excesso para o ano de 1990; ano sugerido como base para os cálculos de emissões pelo Intergovernmental Panel on Climate Change. Em relação à biomassa total calculada pelo autor para aquele ano como desmatada $\left(600,2 \times 10^{6} \mathrm{t}\right)$, este valor representa apenas $0,08 \%$ do geral. Para os tipos de vegetação que representam a maior parte do estoque de carbono na região amazônica, tais como as florestas densas (Db), medidas detalhadas concordam bem com as estimativas feitas a partir dos dados de RADAMBRASIL (e.g., Nacimento \& Laurance, 2002). Embora de pouca expressão numérica no caso das campinas, a discrepância constatada é uma indicação da necessidade de revisão na classificação dos tipos vegetais amazônicos que são usados para os cálculos de emissão de gases do efeito estufa pela troca do uso da terra na Amazônia.

\section{CONCLUSÕES}

Este trabalho aponta novos valores para biomassa acima do solo em tipologias de campinas arborizadas (La) na Amazônia brasileira. Do ponto de vista dos cálculos de emissão de gases pela queima e decomposição da biomassa na região, eles contribuem para reduzir as incertezas em valores atualmente utilizados nas estimativas de biomassa e estoques de carbono que são afetados pela ação do fogo eventual e/ou conversão destes sistemas em usos antropogênicos.

\section{AGRADECIMENTOS}

Este trabalho foi apoiado pelo projeto "Agroecossistemas da Amazônia - Roraima", suportado pelo Convênio 027/99 entre o INPA e o Governo de Roraima. 


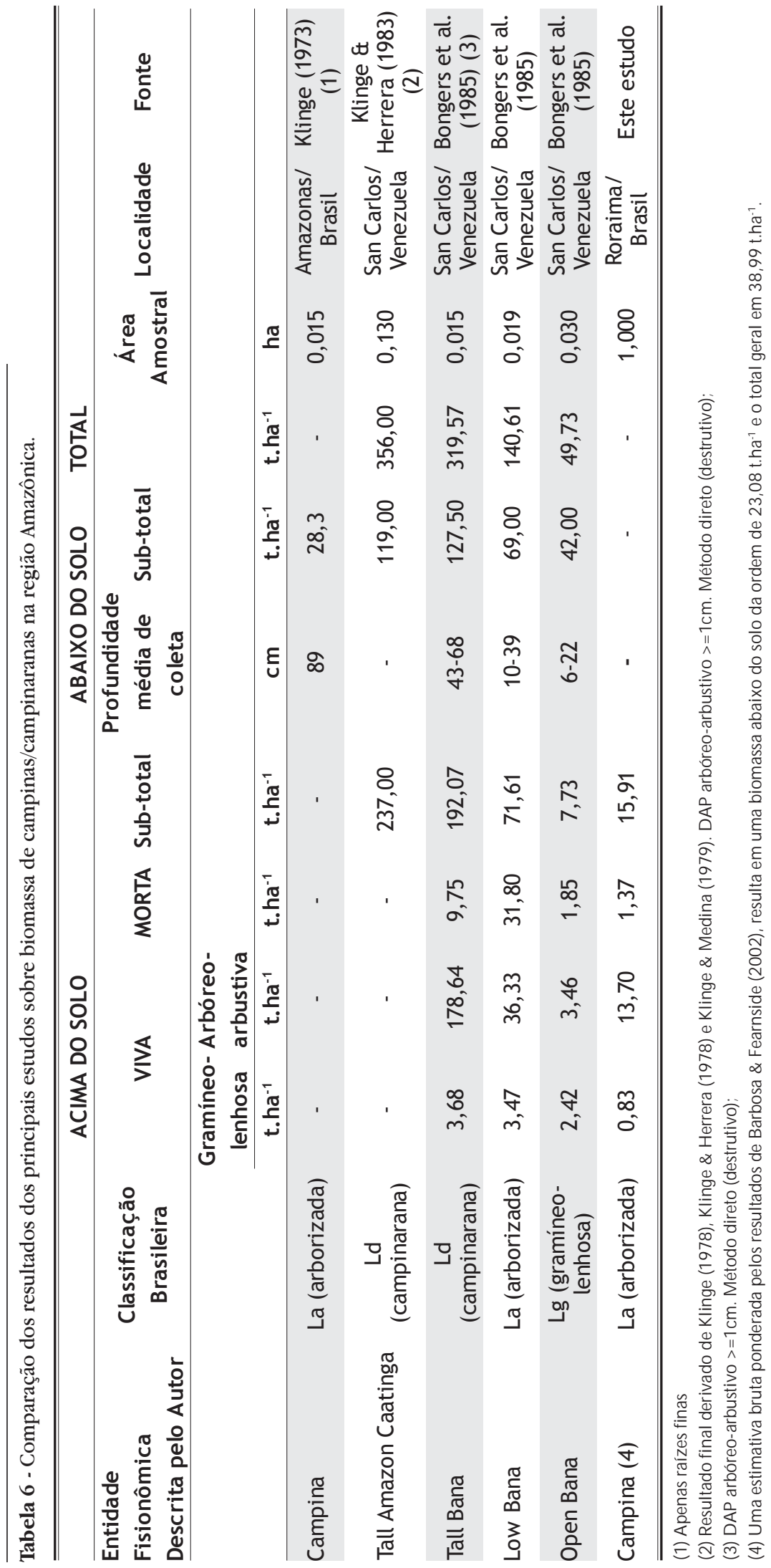

José Lima (INPA/CPBO), José Guedes (INPA/CPBO), Núbia Abrantes (UFRR/ Dep. Biologia), Cice Maduro (Museu Integrado de Roraima) e Herundino Ribeiro do Nascimento Filho (SEPLANRR), colaboraram com a coleta de material em campo. O Dr. Philip Martin Fearnside (INPA/CPEC) contribuiu na revisão do manuscrito.

\section{BIBLIOGRAFIA CITADA}

Alves, D.S.; Soares, J.V.; Amaral, S.; Mello, E.M.K.; Almeida, S.A.S; Silva, O.F.; Silveira, A.M. 1997. Biomass of primary and secondary vegetation in Rondônia, Western Brazilian Amazon. Global Change Biology, 3: 451-461.

Anderson, A .B. 1981. White-sand vegetation of Brazilian Amazonia. Biotropica, 13(3):199-210.

Anderson, A.B.; Prance, G.T.; Albuquerque, B.W.P. 1975. Estudos sobre a vegetação das Campinas Amazônicas - III - A vegetação lenhosa da Campina da Reserva Biológica INPA - SUFRAMA (Manaus - Caracaraí, km 62). Acta Amazonica, 5(3): 225-246.

Barbosa, R.I. 2001. Savanas da Amazônia: emissão de gases do efeito estufa e material particulado pela queima e decomposição da biomassa acima do solo, sem a troca do uso da terra em Roraima, Brasil. Tese de Doutorado, INPA/FUA, Manaus. 212p.

Barbosa, R.I; Fearnside, P.M. 2002. Área e biomassa dos ecossistemas terrestres da Amazônia Legal Brasileira: estimativas para avaliações da emissão de gases do efeito estufa. Instituto Nacional de Pesquisas da Amazônia / Núcleo de Pesquisas de Roraima (INPA/NPRR). 30p. (Relatório de Pesquisa - Biblioteca do INPA, Manaus).

Bongers, F.; Engelen, D.; Klinge, H. 1985. Phytomass structure of natural plant communites on spodosols in southern Venezuela: the Bana woodland. Vegetatio, 63: 13-34.

Brasil 1975. Projeto RADAMBRASIL Levantamento dos Recursos Naturais (Volume 8). Ministério das Minas e Energia, Departamento Nacional da Produção Mineral. 427p.

Brower, J.E.; Zar, J.H.; von Ende, C.N. 1998. Field and laboratory methods for general ecology ( $4^{\mathrm{a}}$ Ed.). Boston, WCB-McGraw-Hill. 273p. 


\section{ACTA AMAZONICA}

BIOMASSA ACIMA DO SOLO DE UM ECOSSISTEMA DE "CAMPINA"

EM RORAIMA, NORTE DA AMAZÔNIA BRASILEIRA
Brown, S. 1997. Estimating biomass and biomass change of tropical forests. FAO Forestry Paper 134. 55p.

Brown, S.; Lugo, A. 1992. Aboveground biomass estimates for tropical moist forests of Brazilian Amazon. Interciencia, 17(1): 8-18.

Eiten, G. 1992. Natural Brazilian vegetation types and their causes. Anais da Academia Brasileira de Ciências, 64 (Supl. 1): 35-65.

Fearnside, P.M. 1992. Forest biomass in Brazilian Amazônia: Comments on the estimate by Brown and Lugo. Interciencia, 17(1): 19-27.

Fearnside, P.M. 1994. Biomassa das florestas amazônicas brasileiras. In: Anais do Seminário Emissão x Seqüestro de $\mathrm{CO}_{2}$ Uma Oportunidade de Negócios para o Brasil. Companhia Vale do Rio Doce, Rio de Janeiro. pp. 95-124.

Fearnside, P.M. 1997. Greenhouse gases from deforestation in Brazilian Amazonia: net committed emissions. Climatic Change, 35(3): 321-360.

Ferreira, C.A.C. 1997. Variação florística e fisionômica da vegetação de transição campina, campinarana e floresta de terra firme na Amazônia Central, Manaus (AM). Dissertação de Mestrado, Universidade Federal Rural de Pernambuco. 112p.

Higuchi, N.; Santos, J.; Ribeiro, R.J.; Minette, L.; Biot, Y. 1998. Biomassa da parte aérea da vegetação da floresta tropical úmida de terra-firme da Amazônia Brasileira. Acta Amazonica, 28(2): 153-166.

IBGE 1992. Manual Técnico da Vegetação Brasileira. Série Manuais Técnicos em Geociências 1. IBGE, Rio de Janeiro. 92p.

INPE 2002. Monitoramento da floresta Amazônica brasileira por satélite 2000-2001. Ministério da Ciência e Tecnologia/ Instituto Nacional de Pesquisas Espaciais. 21p.

Jordan, C.F.; Uhl, C. 1978 Biomass of a terra firme forest of the Amazon Basin. Oecol. Plantar., 13: 387-400.

Klinge, H. 1973. Root mass estimation in lowland tropical rain forests of Central Amazonia, Brazil. I . Fine root masses of a pale yellow latosol and a giant humus podzol. Tropical Ecology, 14(1): 29-38.

Klinge, H. 1978. Studies on the ecology of Amazon Caatinga forest in southern Venezuela. 2 . Biomass dominance of selected tree species in the Amazon Caatinga near San Carlos de Rio Negro. Acta Cientifica Venezolana, 29: 258-262.

Klinge, H.; Herrera, R. 1978. Biomass studies in Amazon Caatinga forest in southern Venezuela. I. Standing crop of composite root mass in selected stands. Tropical Ecology, 19(1): 93-106.

Kllinge, H.; Herrera, R. 1983. Phytomass structure of natural plant communities on spodosols in southern Venezuela: the Tall Amazon Caatinga forest. Vegetatio, 53: 65-84.
Klinge, H.; Medina, E. 1979. Rio Negro Caatingas and Campinas, Amazonas States of Venezuela and Brazil. In: R. L. Specht (ed.), Ecosystems of the World: Heathlands and Related Shrublands. Amsterdan, Elservier Scientific Pub., pp. 483-488.

Lu, D.; Mausel, P.; Brondizio, E.; Moran, E. 2002. Above-ground biomass estimation of successional and mature forests using TM images in the Amazon Basin. In: Symposium on Geospatial Theory, Processing and Applications. Ottawa, Canada (www.isprs.org/commission4/proceedings/pdfpapers/059.pdf).

MCT 2002. Emissões de gases do efeito estufa da queima de biomassa no Cerrado não-antrópico utilizando dados orbitais - Primeiro Inventário Brasileiro de Emissões Antrópicas de Gases do Efeito Estufa. Ministério da Ciência e Tecnologia, Coordenação Geral de Mudanças Globais. Brasília-DF. 53p.

Mourão, M.; Xaud, H.A.M.; Arco-Verde, M.F.; Tonini, H.; Moura Neto, M.A. 2003. Precipitação pluviométrica em áreas de transição savana-mata de Roraima: campos experimentais Serra da Prata e Confiança. Embrapa Roraima. Comunicado Técnico (no prelo).

Nascimento, H.; Laurance, W.F. 2002. Total aboveground biomass in central Amazonian rainforest: A landscape-scale study. Forest Ecology and Management, 168:. 311-321.

Overman, J.P.M.; Witte, H.J.L.; Saldarriaga, J.G. 1994. Evaluation of regression models for above-ground biomass determination in Amazon rainforest. Journal Tropical Ecology, 10: 207-218.

Prance, G.T. 1975. Estudos sobre a vegetação das Campinas Amazônicas. I . Introdução a uma série de publicações sobre a vegetação das Campinas Amazônicas. Acta Amazonica 5(3): 207-209.

Rodrigues, W.A. 1961. Aspectos fitossociológicos das catingas do Rio Negro. Boletim do Museu Paraense Emílio Goeldi (Nova Série Botânica), 15: 1-41.

Sioli, H. 1960. Estratificação radicular numa caatinga baixa do Alto Rio Negro. Boletim do Museu Paraense Emílio Goeldi (Nova Série Botânica), 10: 1-9.

Takeuchi, M. 1960. A estrutura da vegetação na Amazônia III - A mata de campina na região do Rio Negro. Boletim do Museu Paraense Emílio Goeldi (Nova Série Botânica), 8: 1-13. 